\title{
COMPUTATIONAL STUDY OF FLOW AND HEAT TRANSFER WITH ANTI CROSS- FLOWS (ACF) JET IMPINGEMENT COOLING FOR DIFFERENT HEIGHTS OF CORRUGATE
}

\author{
Radheesh Dhanasegaran \\ Chalmers University of Technology \\ Gothenburg, Sweden
}

\author{
Ssheshan Pugazhendhi \\ Indian Institute of Technology Madras (IITM) \\ Chennai, TamilNadu, India
}

\section{ABSTRACT}

In the present study, a flow visualization and heat transfer investigation is carried out computationally on a flat plate with 10x1 array of impinging jets from a corrugated plate. This corrugated structure is an Anti-Cross Flow (ACF) technique which is proved to nullify the negative effects of cross-flow thus enhancing the overall cooling performance. Governing equations are solved using k- $\omega$ Shear Stress Transport (SST) turbulence model in commercial code FLUENT. The parameter variation considered for the present study are (i) three different heights of $A C F$ corrugate $(C / D=1,2 \& 3)$ and (ii) two different jet-to-target plate spacing $(\mathrm{H} / \mathrm{D}=1 \& 2)$. The dependence of ACF structure performance on the corrugate height (C/D) and the flow structure has been discussed in detail, therefore choosing an optimum corrugate height and visualizing the threedimensional flow phenomena are the main objectives of the present study. The three-dimensional flow separation and heat transfer characteristics are explained with the help of skin friction lines, upwash fountains, wall eddies, counter-rotating vortex pair (CRVP), and plots of Nusselt number. It is found that the heat transfer performance is high at larger corrugate heights for both the jet-to-plate spacing. Moreover, the deterioration of the skin friction pattern corresponding to the far downstream impingement zones is greatly reduced with ACF structure, retaining more uniform heat transfer pattern even at low H/D values where the crossflow effects are more dominant in case of the conventional cooling structure. In comparison of the overall heat transfer performance the difference between $\mathrm{C} / \mathrm{D}=3 \& \mathrm{C} / \mathrm{D}=2$ for $\mathrm{H} / \mathrm{D}=2$ is significantly less, thus making the later as the optimal configuration in terms of reduced channel height.

Keywords: Cross flow, Flow Visualization, Jet Impingement, Heat Transfer
NOMENCLATURE

\begin{tabular}{|ccc|}
\hline \multicolumn{1}{|c}{ Alphabets } & Abbreviations & Subscripts \\
C-Corrugate & ACF-Anti-Cross & x-span-wise direction \\
Height, [m] & Flows & \\
D-Impingement & AL-Attachment line & y-normal direction \\
Hole Diameter, & & \\
[m] & & \\
H-Jet-to-target & CRVP-Counter & z-streamwise \\
plate spacing, & Rotating Vortex & direction \\
[m] & Pair & \\
P-Hole Spacing, & NA-Nodal point of & \\
{$[\mathrm{m}]$} & Attachment & \\
& NS-Nodal point of & \\
& Separation & \\
& Nu-Nusselt number \\
& SP-Saddle point \\
\hline
\end{tabular}

\section{INTRODUCTION}

The necessity of increased turbine inlet temperatures in the modern air breathing engines substantiates the design of efficient cooling systems for these components. Hot spot formations due to the hot temperature mainstream lead to thermal failure of the blade materials. A combination of different cooling methodologies such as film cooling for the external surface and impingement cooling for the internal surface are generally used especially in the first stages of turbines (NGV \& HPT). The later method is mostly found in the regions where localized heat transfer is necessary and that cannot be protected by the film cooling holes. In impingement cooling, crossflow from the upstream jets is one of the major factor that may affect the heat transfer performance. This crossflow is sometimes useful in providing localized heat 
transfer augmentation but mostly deflects the downstream jets thus making them less significant.

The earlier studies on this crossflow started with the experimental investigations of Metzeger \& Korstad [1], which concluded that the crossflow reduces average heat transfer. The studies of Florschuetz et al. [2], included the significance of considering initial crossflow for jet impingement array especially in simulating the impingement cooled mid-chord region of a gas turbine airfoil. A similar study of considering initial crossflow effects has been carried out by Chambers et al. [3] in which they showed that the initial crossflow strongly influenced the heat transfer distribution by comparing different mass flux ratios between the crossflow and jet.

It is not only the flow parameters but also the geometric configuration that influences the heat transfer performance. Therefore, this parametric study has its inevitable significance. Many such studies from the beginning of $1980^{\circ}$ s with the works of Florschuetz et al. [4] in which they investigated the effects of geometrical parameters like hole spacing, hole patterns and channel heights normalized by the hole diameter. Similar things can be understood from the open literature. Ramakumar and Prasad [6] performed computational investigations on a concave surface imitating the leading-edge impingement cooling for inter-jet spacing \& target plate spacing thus explaining the heat transfer using flow visualization results.

In the recent years combined effects of geometrical variations and crossflow have been examined. Such studies were evident from Lee et al. [10], where they analyzed the crossflow effects for staggered denser hole array spacing. Their studies concluded that the accumulated crossflows in the impingement channels caused detrimental effects to the downstream jets by reducing their peak Nusselt number values for smaller hole spacing initially but reverses for normalized streamwise distances greater than 30 due to the enhanced mixing between coolant and accumulated crossflow thus increase in turbulent transports. This local augmentation of Nusselt number occurs for crossflows at low Reynolds number values which is again explained by Lee et al. [11].

For a low jet-to-target plate spacing $(\mathrm{H} / \mathrm{D}=2)$, the initial crossflow effects were studied by Way-hayee et al. [12] both experimentally and numerically for different crossflow velocities. Their results concluded that the peak Nusselt number value increases with increase in crossflow velocities. Xing and Weigand [9] in their experimental studies has considered a range of Reynolds number to carry out a study for different crossflow schemes and concluded that jet-to-plate spacing (H/D) value of 3 as the optimum jet-to-plate spacing. A novel impingement cooling structure known as the Anti-Cross Flows (ACF) to negate the ill-effects of crossflows has been was introduced by Chi et al. [8]. Their conclusions provided positive results for wall temperature reduction compared to the conventional cooling structure.

The present study investigates the dependence of the ACF geometry on height of the corrugate at lower target plate spacing $(\mathrm{h} / \mathrm{d}=1 \& 2)$. Also, the heat transfer dependence on the topology of flow structure has not been very well explained by the past literature. Therefore, a numerical flow visualization study has been carried out for different heights of corrugate on a 10x1 ACF impingement domain in this work.

\section{COMPUTATIONAL METHODOLOGY}

The physical model consists of a flat plate separated by a distance that is non-dimensionalized with respect to the impingement hole diameter as H/D. There are 10x1 jets impinging normal to a flat plate from a corrugated nozzle plate. Three different heights of the corrugate $(C / D=1,2 \& 3)$ are the parameter to be investigated for two different jet-to-target plate spacing $(\mathrm{H} / \mathrm{D})$ of $1 \& 2$. The streamwise and the span-wise pitches $\left(\mathrm{P}_{\mathrm{z}} / \mathrm{D} \& \mathrm{P}_{\mathrm{x}} / \mathrm{D}\right)$ are maintained to be a constant value of 4 and 8 respectively. To keep this spanwise pitch, a symmetric boundary condition has been imposed on the side walls. The impingement hole diameter (D) will also be kept a constant value of $0.005 \mathrm{~m}$ for the entire study.

A finite volume grid generation tool GAMBIT 2.4.6 was used to generate the background grid, where a multi-block grid generation methodology has been used to create a structured for a computational efficient grid. A very fine boundary layer (B.L) mesh is used near the wall regions to capture the flow phenomena more precise. Hexahedron elements are used for the mesh generation of the computational domain shown in the figure 1. After performing mesh-independence studies, a final grid size containing approximately 5.2 million cells were used for all the computations.

A velocity inlet condition is given for the impingement holes where the coolant is supplied at an ambient condition of $293 \mathrm{~K}$ at $32 \mathrm{~m} / \mathrm{s}$. The target plate is maintained at a constant wall temperature of $373 \mathrm{~K}$, so that a temperature difference of $80 \mathrm{~K}$ is maintained between the incoming jet from the impingement holes and the target plate. The flow Reynolds number is approximately 11000. The governing equations are solved using k- $\omega$ Shear Stress Transport (SST) turbulence model in commercial code FLUENT model after practicing validation with the published experimental works of Esposito et al [7] and Chi et al [8]. The validation plot in figure 2, shows the comparison of spanwise averaged $\mathrm{Nu}$ for $\mathrm{H} / \mathrm{D}-1 \mathrm{C} / \mathrm{D}-3$ with the experimental data of Chi et al. (2013). The peak and minimum values are slightly over and under predicted which is even the scenario in the computational works of Chi et al. [6]. Otherwise it has a good overall agreement.

All the equations are discretized with second order accurate upwind scheme in space and SIMPLE algorithm is used for pressure-velocity coupling. A convergence criteria of the order of 1e-05 has been set for the residual calculation along with the monitors of Area Weighted Average value of surface heat flux. 


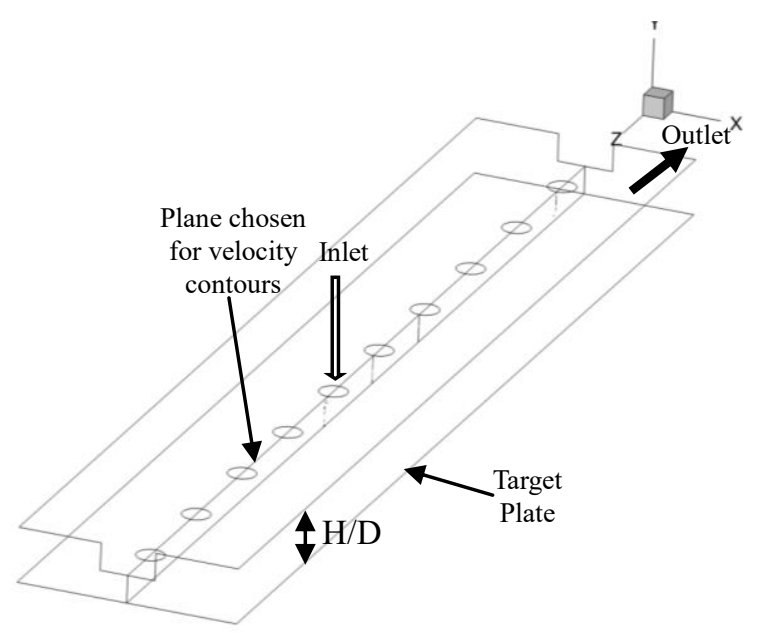

$50 \mathrm{D}$

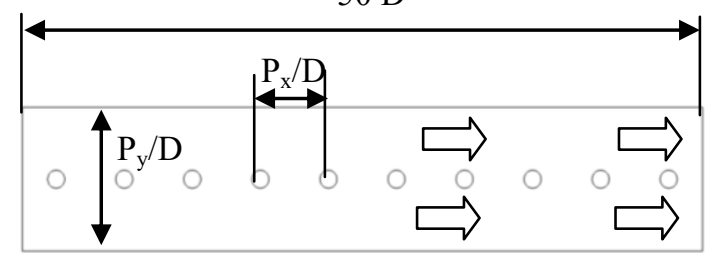

$\square \quad$ Direction of Crossflow/Outflow

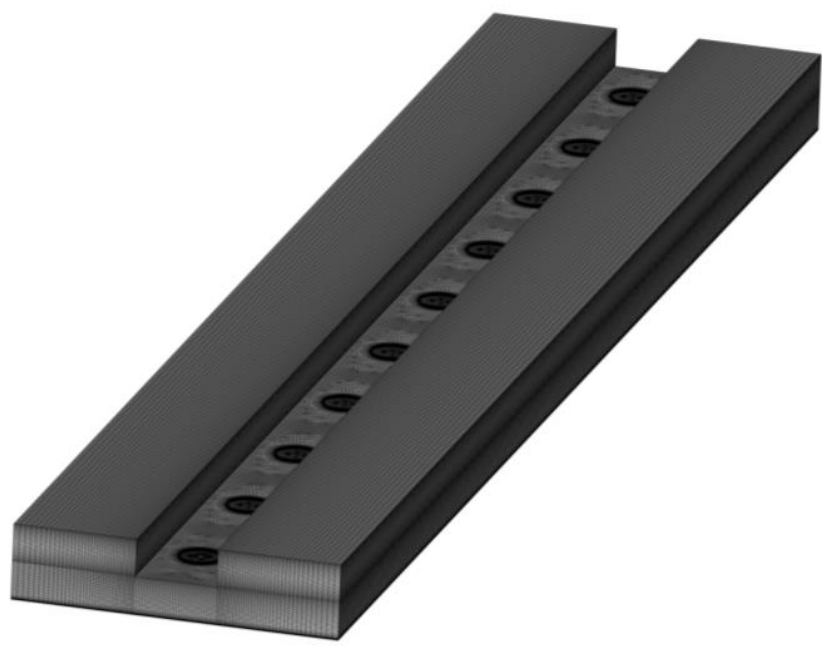

Figure 1. Geometric Details \& Mesh

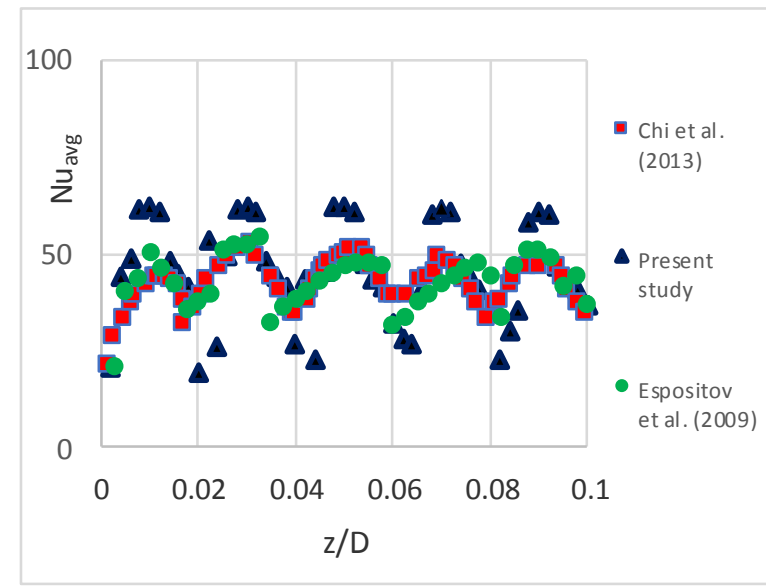

Figure 2. Validation

\section{RESULTS \& DISCUSSION}

When the jet impinges on the target plate, a stagnation point is created, which is the nodal point of attachment on the surface. The flow separation occurs at this location by the shearing action of the fluid on the target plate surface. Corresponding to the different regions in these skin-friction line pattern the heat transfer pattern changes. Therefore, changes observed in the skin-friction pattern is explained to correlate the conforming heat transfer variation in the following sub sections.

\section{Topology of Flow \& Heat Transfer Variation}

The upwash fountains and skin-friction lines are used in explaining the physics of flow and heat transfer.

\section{$H / D=1$}

Since the target plate spacing is small for $H / D=1$, the jet strikes the target plate before becoming a fully developed flow. Therefore, the existence of potential core can be utilized in providing high values of heat transfer coefficients corresponding to the stagnation zones which because of the high momentum present in it. For $\mathrm{C} / \mathrm{D}=3$, the deflection of the downstream jets has been greatly reduced compared to $\mathrm{C} / \mathrm{D}=1 \& 2$. This deflection results from the lower channel height thus the flow washes down. The upwash fountains are more prominent for $\mathrm{C} / \mathrm{D}=3$. For lower corrugate heights, the existence of relatively small vortical structures just ahead the far downstream jets, results in peak heat flux values which can be seen from the figures $3.1,3.2 \& 3.3$. Also, the diminishing skin-friction pattern in these far impingement zones are significantly reduced for $\mathrm{C} / \mathrm{D}=3$ that provides more uniform heat transfer values. The upwash flow occurs from the saddle points (SP) and impinges on the top wall where low velocity wall jet is formed. The entrainment of the top wall jets into the main jet results in CRVP formation. 
The figure 5(a) presents the upwash flow in the spanwise direction for the last row downstream jet where phenomenal vortex breakdown occurs resulting in the formation of a smaller vortex below the wall eddies being an effect of the corrugate structure. As the corrugate height is increased, the top wall jet near the orifice diminishes. Therefore, deterioration in the skinfriction decreases as the corrugate height increases.

\section{$H / D=2$}

As $\mathrm{H} / \mathrm{D}$ increases, the decrease in jet momentum causes the width of the potential core to shrink. The wall eddies are absent due to the reduced movement of the upwash towards the top wall. As moved towards the downstream jets, the upwash decreases in intensity and the flow becomes smooth. This is because of the crossflow effects forming a vortex immediately before each jet in the far downstream locations. With the increase in corrugate height, the jet deflection decreases and the potential core broadens. The heat transfer pattern also becomes uniform with reduction in skin-friction pattern deterioration in these far downstream jet impingement zones. Here, the upwash flow arises from the nodal point of separation (NS).

The spanwise upwash pattern is shown in figure 5(b). Like $\mathrm{H} / \mathrm{D}=1$, vortex breakdown occurs due to the corrugate geometry leading to the top wall jet interaction into the mainstream jet thus pulling it away from the wall. Secondary stagnation zones are formed between the large vortex and the target plate that gives enhanced heat transfer values in the spanwise direction.

\section{Nusselt Number Variation}

From the plots of streamwise centerline Nusselt number variation along the streamwise direction shown in figures $6 \& 7$, it can be observed that the lower corrugate heights of $H / D=1$ has peak values greater than $\mathrm{C} / \mathrm{D}=3$, corresponding to the impingement zones. While considering the regions between NA and $A L, C / D=3$ has better values of Nusselt number than others. But for $\mathrm{H} / \mathrm{D}=2$, even in the impingement zones and zones in between streamwise $\mathrm{Nu}$ values are higher than the other cases.

The spanwise averaged plots of Nusselt number presented in figure 8 shows that the H/D-2 C/D-2 \& H/D-3 have highest overall values and the existence of secondary peaks is due to the strong attachment lines (AL). To compare the overall performance, the area weighted average (AWA) of surface Nusselt number is plotted in figure 9 for all the cases. This shows that H/D-2 C/D-2 \& C/D-3 have less than $1 \%$ difference.

The figures presented below are in the order of contours of velocity, upwash flow, heat flux and zoomed right side view of upwash.
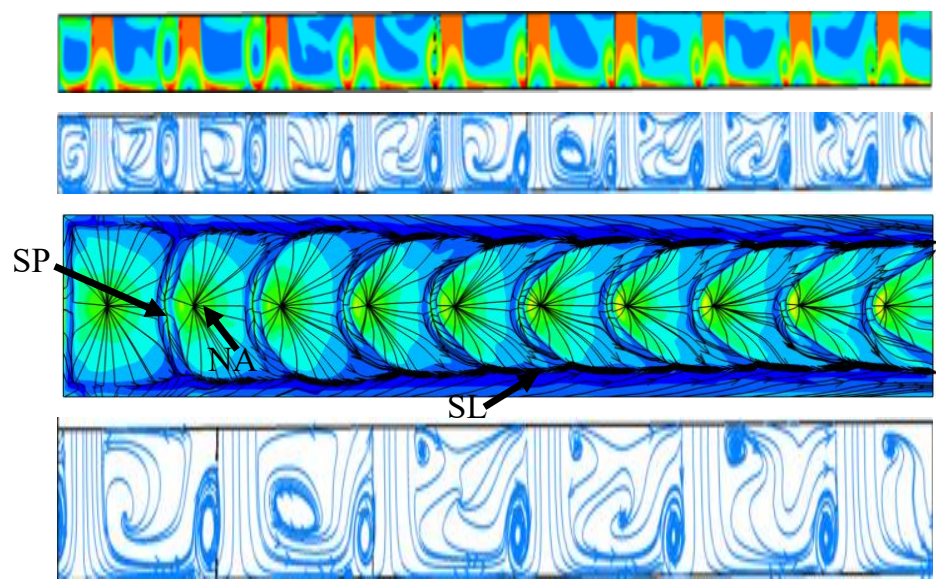

Figure 3.1. H/D-1 C/D-1
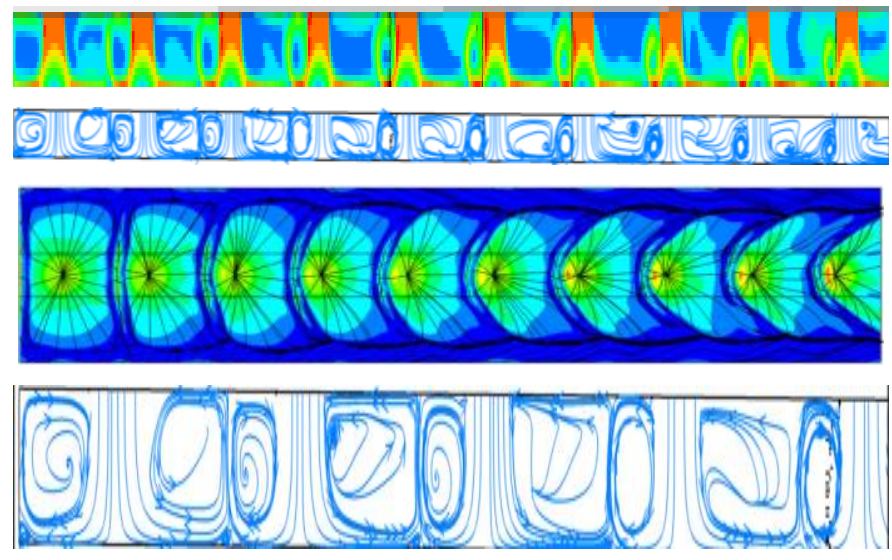

Figure 3.2. H/D-1 C/D-2
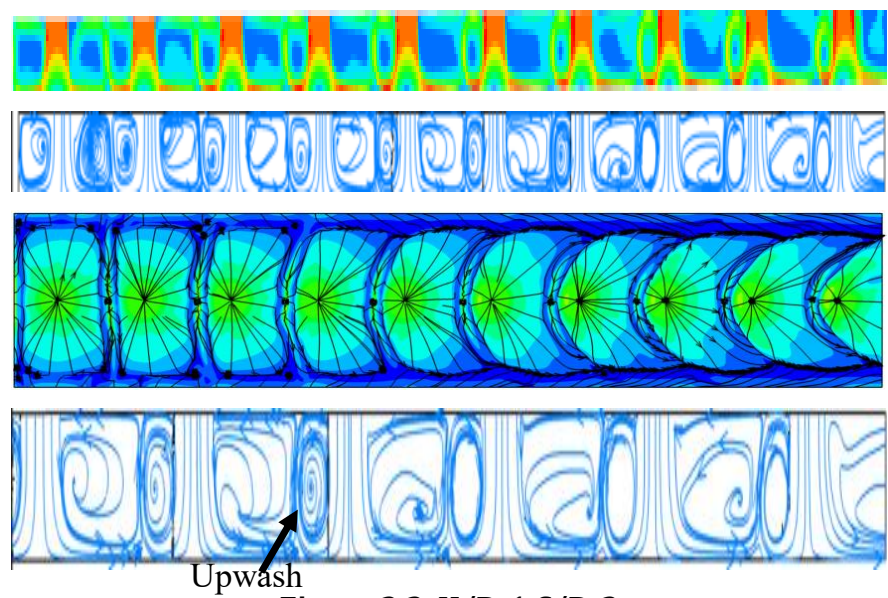

Figure 3.3. H/D-1 C/D-3

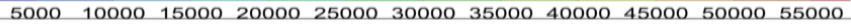

Figure 3.4. Legend for Heat Flux Contours 

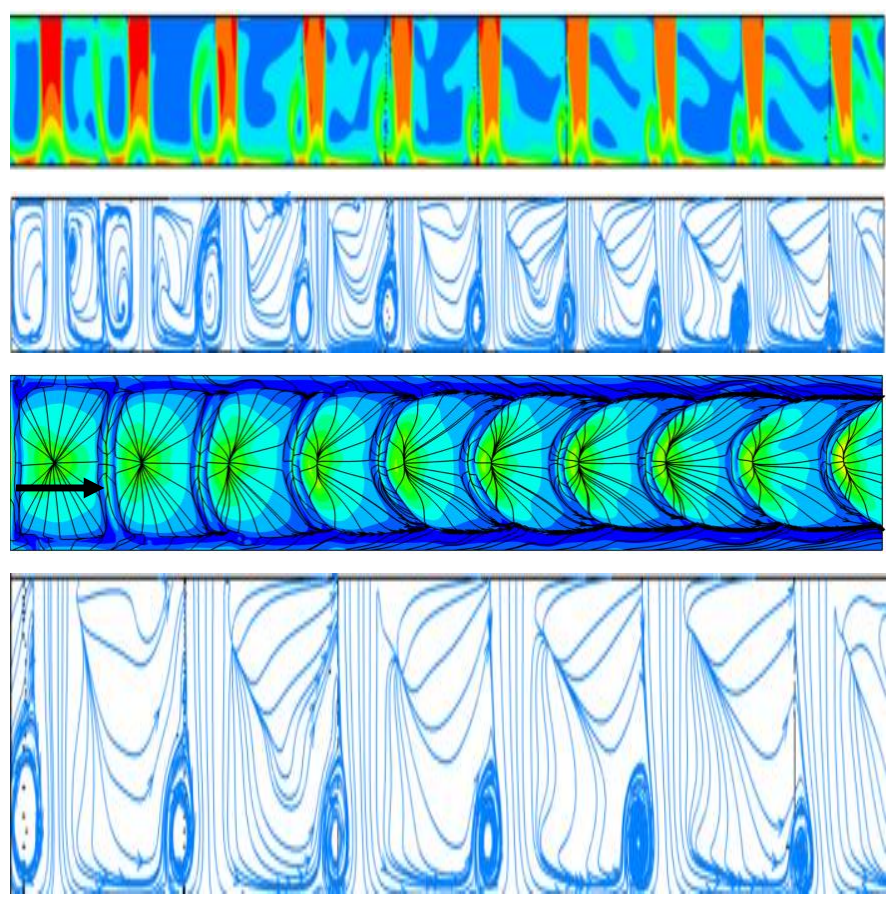

Figure 4.1. H/D-2 C/D-1
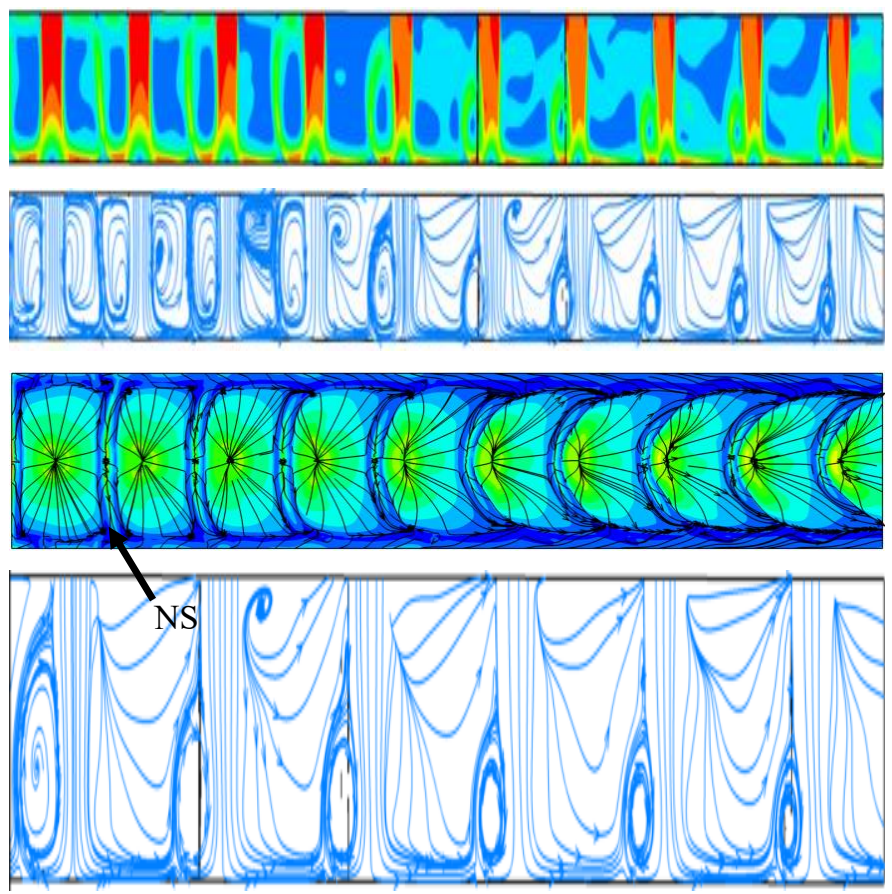

Figure 4.2. H/D-2 C/D-2
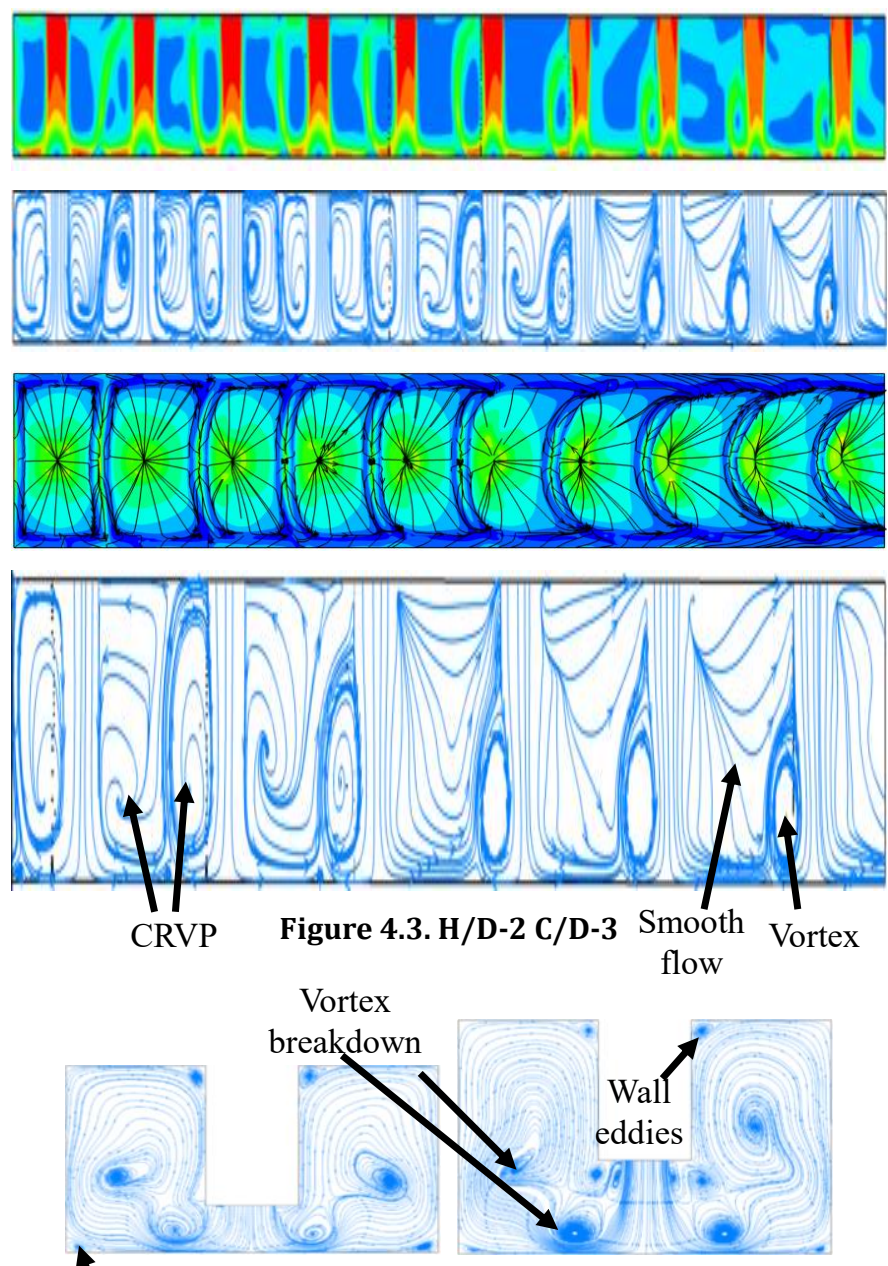

1

(a) H/D-1 C/D-3

(b) H/D-2 C/D-3

Figure 5. Upwash flow in Spanwise direction Secondary stagnation

zone

Streamwise Nu Comparison for H/D=1

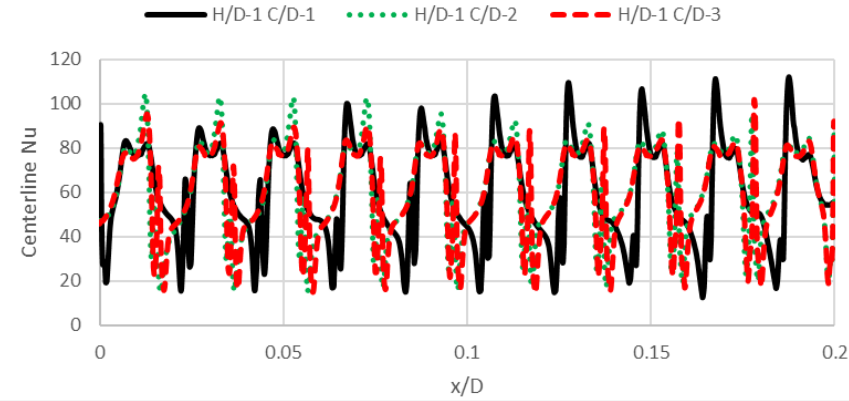

Figure 6. Comparison of Streamwise Nusselt number for $\mathrm{H} / \mathrm{D}=\mathbf{1}$ 


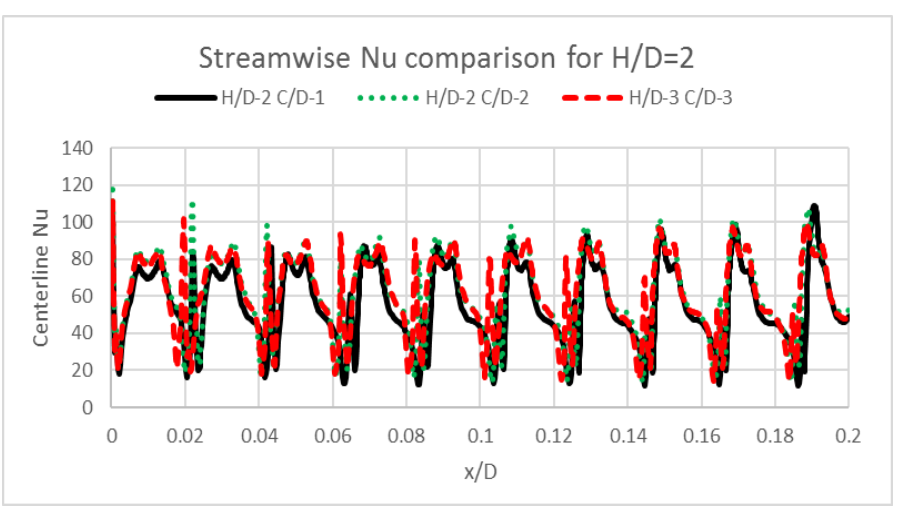

Figure 7. Comparison of Streamwise Nusselt number for

$$
H / D=2
$$

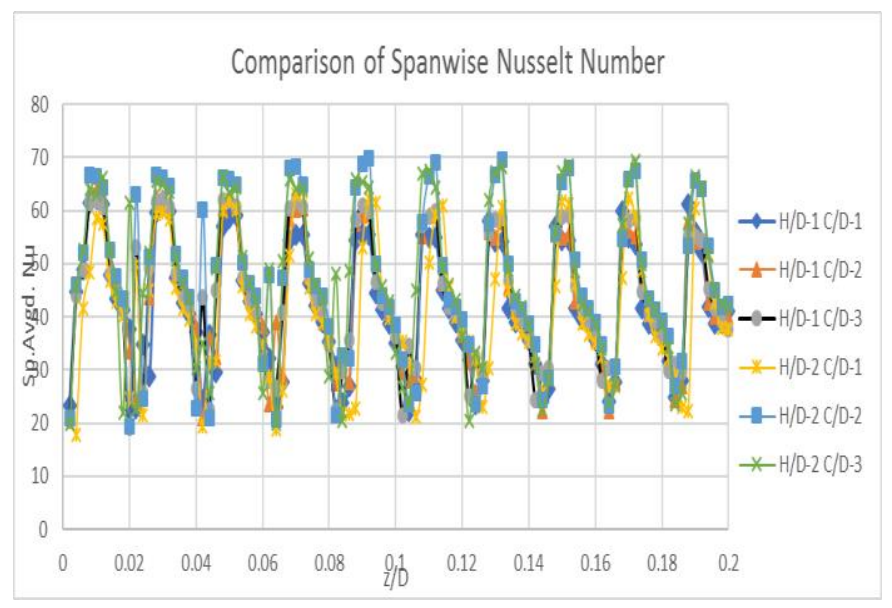

Figure 8. Comparison of Spanwise Averaged Nusselt

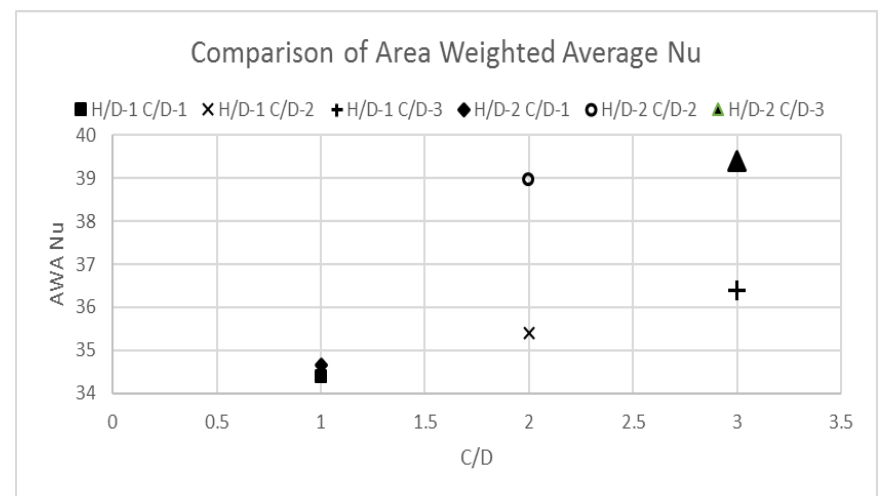

Figure 9. Comparison of Area Weighted Average Nusselt numbers

\section{CONCLUSIONS}

A flow visualization and heat transfer study has been conducted on a 10x1 array of jets emanating from a corrugated plate to minimize the effects of crossflow for three different corrugate heights $(C / D=1,2 \& 3)$ for each $H / D=1 \& 2$. The heat transfer characteristics are explained with the 3D flow separation techniques such as upwash flow and surface flow visualization by skin-friction lines and Nusselt number variation. The following conclusions have been observed.

(i) For both $\mathrm{H} / \mathrm{D}(=1 \& 2)$, the presence of upwash is more prominent at higher $\mathrm{C} / \mathrm{D}$ values which marks the decreasing strength of the crossflow with increasing corrugate heights.

(ii) The skin-friction pattern deterioration also decreases with increasing corrugate heights providing a uniform heat transfer pattern for each impingement zones.

(iii) The streamwise Nusselt number variations predicts local peak values are higher for the far downstream impingement zones in lower $\mathrm{C} / \mathrm{D}$ values for $H / D=1$ but is subjugated by higher $C / D$ values for $H / D=2$. This shows that the crossflow effect is reduced for $H / D=2$ especially for $C / D=2$ $\& \mathrm{C} / \mathrm{D}=3$. The overall comparison of area weighted average values of $\mathrm{Nu}$ also proves it and the difference between them is less than $1 \%$ thus making $\mathrm{H} / \mathrm{D}=2 \mathrm{C} / \mathrm{D}=2$ an optimum value in terms of reduced channel height than $H / D=2 C / D=3$.

Therefore, $\mathrm{H} / \mathrm{D}=2 \quad \mathrm{C} / \mathrm{D}=2$ serves as an optimal configuration for Anti-Cross Flows (ACF) cooling structure in consideration of the cases dealt in the present study.

\section{ACKNOWLEDGMENTS}

The authors acknowledge the people of CFD-Centre (Turbomachines Laboratory) at IIT Madras, Chennai, India for their kind support in providing computational facilities for running the simulations and Dr. Rajesh Kumar Panda (Senior Engineer, Powergrid Corporation, India) for his valuable mentorship.

\section{REFERENCES}

[1] Metzger. D. E and Korstad. R. J, "Effects of Crossflow on Impingement Heat Transfer", Journal of Engineering for Power, 1972.

[2] Florschuetz. L. W and Truman. C. R and Metzger. D. E, "Streamwise Flow and Heat Transfer Distributions for Jet Array Impingement with Crossflow", Gas Turbine Conference \& Products Show, 1981.

[3] Florschuetz. L. W, Metzger. D. E and Su. C. C, "Heat Transfer Characteristics for Jet Impingement with Initial Crossflow", ASME Journal of Heat Transfer, 1983. 
[4] Chambers. A. C, Gillespie. D. R. H, Ireland. P. T and Dailey. G. M, "The Effect of Initial Cross Flow on the Cooling Performance of a Narrow Impingement Channel", ASME Journal of Heat Transfer, 2005.

[5] Zuckerman. N and Lior. N, ,Jet Impingement Heat Transfer: Physics, Correlations, and Numerical Modelingee, Advances in Heat Transfer, Volume 39, 2006.

[6] Rama Kumar. B. V. N and Prasad. B. V. S. S. S, "Computational flow and heat transfer of a row of circular jets impinging on a concave surface", Springer Heat and Mass Transfer Journal, 2007.

[7] Esposito. E. I, Ekkad. S. V, Kim. Y and Dutta. P, ,Novel Jet Impingement Cooling Geometry for Combustor Liner Backside Coolingee, ASME Journal of Thermal Science and Engineering Applications, 2009

[8] Chi. Z, Kan. R, Ren. J and Jiang. H, "Experimental and numerical study of the anti-crossflows impingement cooling structure", International Journal of Heat and Mass Transfer, Elsevier, 2013.

[9] Xing. Y and Weigand. B, "Optimum Jet-to-Plate Spacing of Inline Impingement Heat Transfer for Different Crossflow Schemes", ASME Journal of Heat Transfer, 2013.

[10] Lee. J, Ren. Z, Ligrani. P, Lee. D. H, Fox. M. D and Moon. H. K, "Cross-flow effects on impingement array heat transfer with varying jet-to-target plate distance and hole spacing", International Journal of Heat and Mass Transfer, Elsevier, 2014.

[11] Lee. J, Ren. Z, Ligrani. P, Lee. D. H, Fox. M. D and Moon. H. K, "Crossflows from jet array impingement cooling: Hole spacing, target plate distance", Reynolds number effects, International Journal of Heat and Mass Transfer, Elsevier, 2014.

[12] Hayee. M.W Tekasakul. P, Eiamsa-ard. S and Nuntadusit. C, "Effect of cross-flow velocity on flow and heat transfer characteristics of impinging jet with low jet-to-plate distance", Springer Journal of Mechanical Science and Technology, 2014. 